THE G $\alpha q$ ISOFORM OF HETEROTRIMERIC G PROTEIN AND THE PHOSPHOLIPASE C 34 ARE REQUIRED FOR CLIMBING FIBER SYNAPSE ELIMINATION DURING POSTNATAL CEREBELLAR DEVELOPMENT.

MASANOBU KANO1, KOUICHI HASHIMOTO1,2, STEFAN OFFERMANNS ${ }^{3}$, MASAHIKO WATANABE 4 , HIDEO KURIHARA4, HUIPING JIANG5, YANPING WU5, KI-SU JUN5, YOSHIRO INOUE4, HEE-SUP SHIN6, and DIANQING WU5, MELVIN I. SIMON 3

1 Lab. for Neuronal Signal Transduct., Frontier Research Program, RIKEN, Wako, Saitama 351-01, Japan, 2Dept. of Physiol., Jichi Med. Sch., Minamikawachimachi, Tochigi 329-04, Japan, 3Dev. of Biol. 147-75, California Inst. Tech., Pasadena, CA91125, USA., " ${ }^{4}$ Dept. of Anat., Hokkaido Uni. Sch. of Med., Sapporo 060, Japan, 5Dept. of Pharmacol. and Physiol., Univ. of Rochester, Rochester, NY 14642-8711, USA, 6 Dept. of Life Sci., Pohang Univ. of Sci. and Tech.Pohang 790-784, Korea.

In the cerebellum of adult animals, each Purkinje cell is innervated by a single climbing fiber which originate from the inferior olive of the medulla and supply strong excitatory synapses onto proximal dendrites of Purkinje cells. At early developmental stages, however, each Purkinje cell is innervated by multiple climbing fibers. Massive elimination of supernumerary climbing fiber synapses occurs postnatally, and the adult-type one to one innervation pattern is established on around P20. Previous studies indicate that this developmental change of climbing fiber innervation requires the normal formation of excitatory synapses from parallel fibers, the other major excitatory inputs to Purkinje cells. Molecules responsible for this developmental change are largely unknown. In two strains of mutant mice, which are deficient in the $\alpha$-subunit of a GTP-binding protein $\mathrm{Gq}(\mathrm{G} \alpha q)$ and in the $\beta 4$ isoform of phospholipase C (PLCB4), about $40 \%$ of mature Purkinje cells are found to be multiply-innervated by climbing fibers. In contrast, the anatomy of the cerebellum, the morphology of Purkinje cells, and the morphology and density of parallel fiber-Purkinje cell synapses appear normal. Electrophysiological investigation indicated that both climbing fiber- and parallel fiber-Purkinje cell synapses are functional. Developmental changes of climbing fiber innervation during the first and second postnatal weeks are normal. Thus, elimination of multiple climbing fiber innervation that normally occurs during the third postnatal week to attain mono innervation appears to be specifically impaired in the cerebellum of these two mutant mice. These results strongly suggest that Gaq and PLCB4 are key molecules required for the later stage of climbing fiber synapse elimination that occurs during the third postnatal week.

Supported by grants from CREST (Core Research for Evolutional Science and Technology) of Japan Science and Technology Corporation and The Japanese Ministry of Education, Science, Sports and Culture. 


\title{
Abstracts for Posters
}

\section{INVOLVEMENT OF CASPASES IN TNF $\alpha$-MEDIATED OLIGODENDROCYTES CELL DEATH}

\author{
Shin Hisahara ${ }^{*} \dagger$, Shin-ichi Shoji $\dagger$, Hideyuki Okano* ${ }^{*}$ and Masavuki Miura ${ }^{*} \S$ \\ *Department of Neuroanatomy, Biomedical Research Center, Osaka University Medical \\ School, Suita, Osaka 565, Japan, \$CREST (Okano Project), Japan Science and \\ Technology Corporation (JST) and tDepartment of Neurology, Institute of Clinical \\ Medical Sciences, University of Tsukuba, Tsukuba, 1-1-1 Tennoudai, Ibaraki 305, \\ Japan
}

Oligodendrocytes are myelin forming cells in mammalian central nervous system. About $50 \%$ of oligodendrocytes (OLGs) undergo cell death in normal development. In addition, massive OLG cell deaths have been observed in multiple sclerosis (MS). Tumor necrosis factor (TNF) is thought to be one of the mediators responsible for the damage of oligodendrocytes (OLGs). We have examined the involvement of caspase (ICE/CED-3) family in TNF-induced OLG cell death.

Chemical inhibitors of protease activities are powerful tools for studying the involvement of the caspase family in apoptosis. We designed a transient expression system to determine the specificity of benzyloxycarbonyl-Asp- $\mathrm{CH}_{2} \mathrm{OC}(\mathrm{O})-2,6$,dichlorobenzene (Z-Asp-CH2-DCB), an aspartate based-caspase family inhibitor. We administered Z-Asp-CH2-DCB to HeLa cells transfected with caspase-1(ice)-lacZ, caspase-2(nedd2/ich-1L)-lacZ and caspase-11(ich-3)-lacZ. Cell death induced by caspase-1 and caspase-11was greatly reduced by $\mathrm{Z}-\mathrm{Asp}-\mathrm{CH}_{2}-\mathrm{DCB}$, and caspase-2induced cell death was also suppressed but lesser degree. These inhibitory profiles are similar to that of $\mathrm{p} 35$, indicating that Z-Asp- $\mathrm{CH}_{2}-\mathrm{DCB}$ can substitute for $\mathrm{p} 35$. The administration of $\mathrm{Z}$-Asp- $\mathrm{CH}_{2}$-DCB effectively blocked $\mathrm{HeLa}$ cell death induced by TNF- $\alpha / \mathrm{CHX}$. To explore the specificity of protease inhibitors to prevent cell death induced by TNF, TLCK and leupeptin were also administered with TNF- $\alpha / C H X$. The apoptosis of HeLa cells was not prevented by them. These results strongly support the idea that $\mathrm{Z}$-Asp- $\mathrm{CH}_{2}$-DCB could be used as an effective anti-apoptotic reagent in TNF$\alpha$-mediated cell death.

Then we tested the involvement of caspase family in TNF-mediated cell death of OLGs. The addition of TNF- $\alpha$ to primary cultures of OLGs significantly decreased the number of live OLGs in $72 \mathrm{~h}$. DNA fragmentation was detected in TNF-treated OLGs at $36 \mathrm{~h}$ with TUNEL assay. Z-Asp-CH2-DCB protected against the TNF-induced cell death of OLGs. Furthermore, Ac-YVAD-CHO (a specific inhibitor of CASP-1-like proteases) as well as Ac-DEVD-CHO (a specific inhibitor of CASP-3 (CPP32)-like proteases) enhanced the survival of OLGs treated with TNF- $\alpha$, indicating that CASP-1and the CASP-3-mediated cell-death pathway are activated in TNF-induced OLG cell death. RT-PCR analysis showed that purified OLGs express CASP-1, CASP-3 and CASP-11, but not CASP-2. CASP-3 has been detected in PDGF- $\alpha$ positive O-2A progenitor cells and CNPase postive mature OLGs by immunocytochemical analysis. These results suggest that the inhibition of CASP family may be a novel approach to treat neurodegenerative diseases such as MS. 


\title{
DEVELOPMENTALLY-REGULATED PHOSPHORYLATION/ DEPHOSPHORYLATION OF BRAIN MICROTUBULE- ASSOCIATED PROTEIN MAP1B
}

\author{
Emiko Yamauchi ${ }^{\star}$, Kazuyo Muramoto**, Yoichiro Kuroda**, Hisaaki \\ Taniguchi* \\ *Division of Biomedical Polymer Science, Institute for Comprehensive Medical \\ Science, Fujita Health University, Toyoake, Aichi 470-11, Japan, and \\ **Department of Molecular and Cellular Neurobiology, Tokyo Metropolitan Institute \\ for Neuroscience, Fuchu, Tokyo 183, Japan
}

Microtubule-associated protein MAP1B is a major neuronal cytoskeletal protein that is essential for brain development. It is highly expressed in the neonatal brain, but decreases rapidly during synaptogenesis with a concomitant increase of MAP1A, a closely-related microtubule-associated protein. MAP1B undergoes phosphorylation during neuronal differentiation and the states of phosphorylation seem to closely correlate with the brain development.

To characterize the phosphorylation sites of MAP1B, the protein was purified from neonatal rat brain, and analyzed by mass spectrometry. The purified protein was digested with lysyl endoprotease, and the resulting peptide mixture was subjected to liquid chromatography/electrospray mass spectrometric analysis. So far 17 phosphopeptides containing 20 phosphorylation sites have been identified. 9 phosphopeptides were clustered in the middle of the molecule between the microtubule-binding domain and the characteristic heptadeca-repeat domain of unknown function near the C-terminus. Other phosphorylation sites were distributed all over the protein. Phosphopeptides were further analyzed by tandem mass spectrometry to determine the phosphorylation sites. Most phosphorylation sites thus determined were immediately followed by proline, suggesting that MAP1B is one of the major substrate proteins of so-called prolinedirected protein kinases such as MAP kinase and Cdk5-p35. To analyze the regulation of the phosphorylation sites during brain development, similar analyses were carried out with MAP1B purified from rat brains obtained from E18 to P15 rats. Most of the phosphorylation sites were found phosphorylated even in the MAP1B protein purified from E18 brain, when the amount of MAP1B starts to increase. The extent of the phosphorylation did not change significantly up to PO brain. However, two out of the 17 phosphopeptides undergo specific dephosphorylation between $\mathrm{PO}$ and $\mathrm{P} 15$. These results suggest that the phosphorylation of MAP1B occurs at the onset of its expression and that protein is one of the major targets of MAP kinase that is activated through the NGF/trk pathway. The phosphorylation of MAP1B, therefore, is one of the priming steps in the neurite extension. Furthermore, the specific dephosphorylation at the heptadeca repeat domain, which seems to be involved in specific interactions with other proteins, is important for the events during the synaptogenesis. 


\section{Abstracts for Posters}

P44

\section{CELLS, CONTAINING PDGF-ALPHA RECEPTOR MESSAGE ARE DISTINCT FROM THE PLP/DM-20 IRNA-CONTAINING CELLS IN THE EMBRYONIC CNS OF MOUSE.}

Akio Ooba, Anna Ivanova, Kazuhiro Ikenaka.

Natl. Inst. Physiol. Sci., Myodaiji, Okazaki, Aichi 444, Japan.

There is some evidence, that the oligodendrocyte progenitors are present in the embryonic rodent brain very early. Messenger RNAs encoding the alpha receptor for platelet-derived factor (PDGF $\alpha R$ ) and proteolipid protein DM-20 can be detected early in the oligodendrocyte lineage ${ }^{1,2}$. Cells, containing PDGFaRmessage, occupied the ventral rat spinal cord, medulla and a zone within the ventral diencephalon ${ }^{3}$. DM-20+ cells were found in similar locations of the developing mouse $\mathrm{CNS}^{4}$.

The aim of the in situ hybridization studies reported here was to compare the two messages on the adjacent paraffin sections throughout the developing mouse brain. Our results indicate, that the distribution of the PDGF $\alpha \mathrm{R}^{+}$cells in brain is distinct from that of DM-20+ cells. Since both mRNAs are supposed to be present in the cells of the oligodendrocyte lineage, several explanations of this divergence can be proposed. The appearance of these markers might signify the different events in development of oligodendrocyte progenitors. There is always a possibility that PLP and PDGF $\alpha R^{+}$cells might belong to different subpopulations. More detailed analysis about early events in the oligodendrocyte development in vivo is needed, before the relative roles of PLP and PDGF $\alpha \mathrm{R}$ expression in oligodendrocyte lineage can be fully explored.

1. Pringle et al., Development 115 (1992)

2. Ikenaka et al., J. Neurochem. 58 (1992)

3. Pringle and Richardson, Development 117 (1993)

4. Timsit et al., J. Neuroschi. 15 (1995) 


\section{Abstracts for Posters}

P45

\section{DEVELOPMENTAL EXPRESSION OF VOLTAGE-GATED POTASSIUM CHANNELS IN MOUSE CEREBELLAR GRANULE CELLS IN VIVO AND IN THE MICROEXPLANT CULTURE.}

K. Nakahira, Y. Wakazono, R. Shibata, T. Kurahashi, A. Kaneko' and K. Ikenaka. Nat'I Inst. Physiol. Sci., Okazaki 444 Japan, 'Dept. Physiol., Keio Univ. Sch. Med., Tokyo 160 Japan.

In order to elucidate the regulation mechanisms of $\mathrm{K}^{+}$-channel expression during the development of mouse cerebellar granule cells, we examined the expression of $\mathrm{K}^{+}$-channel genes by in situ hybridization. The expression of $\mathrm{Kv4.2}$ mRNA was observed in P7 cerebellum, mainly in the internal granule cell layer. Positive signals were also found in the inner side of external granule cell layer and small cells between internal and external granule cell layer, suggesting that Kv4.2 starts to express in the post-mitotic granule cells. Since Kv4.2 subunit is known to form fast-inactivating $\mathrm{K}^{+}$-channel, we then examined $\mathrm{K}^{+}$currents during the maturation of granule cells using microexplant culture system. First, we identified cerebellar granule cells in this microexplant culture system by immunostaining with the granule cell-specific Zic marker. This staining revealed that granule cells in this culture system migrated radially out from explants and then formed small clusters. The simultaneous recording of their morphology and channel activity were performed by using the recording patch-pipette containing $0.2 \%$ lucifer yellow. Both bipolar and T-shaped/dendrite-bearing cells generated voltage-dependent fast activating potassium currents, however, inactivation of their currents were different. T-shaped cells, which were considered to be mature, showed a fast-inactivating current component. These results suggested that the regulation of specific subunit expression might lead to the electrophysiological maturation. 


\title{
Abstracts for Posters
}

P46

\section{Glutamate transporter (GLAST) as a radial glial marker in primary culture}

\author{
Y. Iwasaki, Y. Otake, M. Watanabe', K. Tanaka ${ }^{2}$, and K. Ikenaka \\ Nat'l Inst. Physiol. Sci., Okazaki 444 Japan, ${ }^{1}$ Hokkaido Univ. Sch. Med., Sapporo \\ 060 Japan, ${ }^{2}$ Nat'I Inst. Neurosci., Kodaira 187 Japan
}

Three types of glutamate transporter have been identified in the mammalian central nervous system. One of them, GLAST, is mainly localized in Bergmann glia in the adult cerebellar cortex and also in the ventricular zone in the developing brain. To characterize the GLAST-expressing cells during early neurogenesis, we examined the expression of GLAST mRNA and proteins in the primary culture prepared from embryonic day 13 (E13) mouse cerebral cortex. GLAST immunoreactivity was observed in E13 plus 1 day in vitro culture (E13+1DIV), increased at E13+3DIV and almost disappeared at E13+7DIV, whereas GFAP immunoreactivity was dramatically increased at E13+7DIV. GLAST was concentrated on the peripheral membrane of cells and at the terminal of processses. Most of $\mathrm{GLAST}^{+}$cells showed bipolar or unipolar shapes which are typical shapes for radial glia. In addition, $\mathrm{GLAST}^{+}$cells were mostly, if not all, also coincided with the cells immunopositive for $\mathrm{RC} 1$ and $\mathrm{RC} 2$ antibodies, the radial glial markers. The localization of GLAST mRNA in E13+1DIV and E13+3DIV culture from mouse cerebral cortex was also examined by in situ hybridization using E13+1DIV and E13+3DIV culture of cerebral cortex, followed by immunostaining with GFAP, RC1 and MAP2 antibodies. GLAST mRNA signals were localizezd mainily in the unipolar or bipolar cells, which coincided to be $\mathrm{GFAP}^{+}$or $\mathrm{RC}_{1}{ }^{+}$cells, but not MAP2 ${ }^{+}$. These results suggest that GLAST is a potent marker for radial glia and is useful to follow the glial cell lineage. 


\section{Abstracts for Posters}

\section{Visualization of dendritic spine formation with GFP-fusion proteins}

Kensuke Hayashi, and Tomoaki Shirao, Department of Neurobiology and Behavior, Gunma University School of Medicine, 3-39-22 Showamachi, Maebashi, 371, Japan

To understand the mechanisms of synaptogenesis, it is critical to identify the mechanisms of the formation of postsynaptic structures as well as those of axonal growth cones. The formation of spines is thought to be a result of stabilization of dendritic filopodia. Spines change their shape during neuronal development, as well as in response to environmental cues. Actin filaments are likely to play an important role in the formation and the shape change of spines.

We have reported that an actin-binding protein, drebrin, accumulates at spines and modulates actin-myosin interaction within spines ${ }^{1,2}$. Drebrin may be one of regulators of the reorganization of actin filaments during formation and the shape change of spines.

To examine which domain of drebrin is responsible for its binding to actin filaments, we transfected $\mathrm{CHO}$ cells with a green fluorescent protein (GFP)cDNA that were fused with various fragments of drebrin-cDNA. The localization of the expressed fusion protein could be observed under a fluorescent microscope without any staining processes. We found that the central region of drebrin (28 a.a.) is important for its binding to actin filaments in $\mathrm{CHO}$ cells.

Next, we introduced the GFP-drebrin fusion cDNA into primary cultured cortical neurons. Two weeks after plating, we found that the length of the dendrites had decreased but the dendritic branching had become enhanced, compared to those of neurons that were transfected with an unfused GFP-cDNA. Three weeks after plating, when synapses were formed between neurons, spines were visible branching off living neurons. The fusion protein was localized at the head of spines. The central region of drebrin, which is responsible for its actin-binding activity, is also necessary for its localization at spines. Spines of neurons with a GFP-drebrin fusion protein were longer than those of GFP-transfected neurons. These results support our hypothesis that drebrin regulates the morphology of dendrites and spines.

We have also found that this spine-visualization system using GFP-cDNA is effective to examine the effect of molecules on dendrite and spine morphogenesis. In particular, it is possible to keep cells alive while monitoring the formation of dendrites and spines using this method.

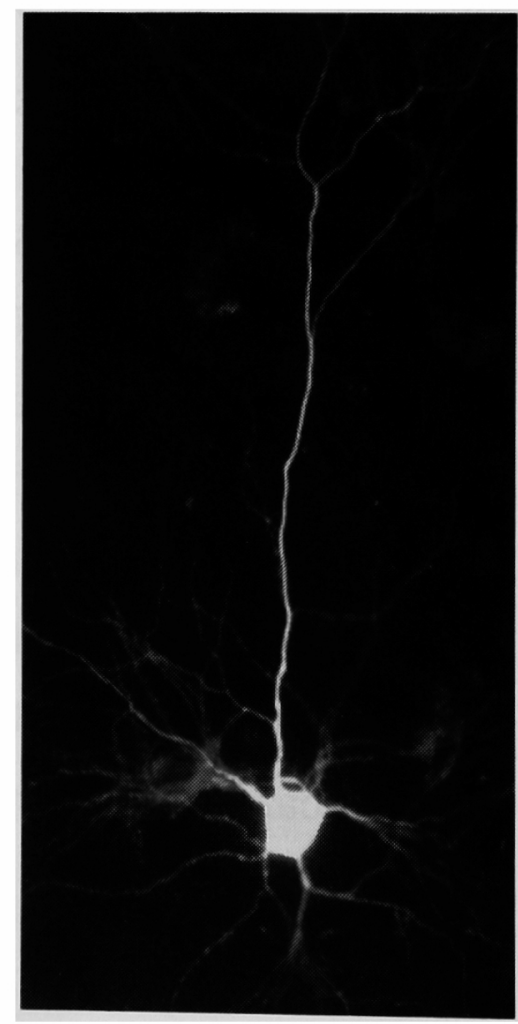

a GFP-transfected neuron

(1)Ishikawa,R., Hayashi,K., et al. (1994) J. Biol. Chem. 47:29928-29933.

(2)Hayashi,K., et al. (1996) J. Neurosci. 16:7161-7170. 


\title{
Abstracts for Posters
}

\section{P48}

\section{CHARACTERIZATION OF TWO cDNAS THAT MAY BE STAGE- SPECIFIC IN THE OLIGODENDROCYTE LINEAGE.}

\author{
C.W. Campagnoni, C.F. Landry, K. Kampf, E.R. Bongarzone, T.M. Pribyl, \\ J.B. Watson and A.T. Campagnoni \\ Mental Retardation Research Center, Neuropsychiatric Institute and Brain Research \\ Institute, UCLA Medical School, Los Angeles, CA 90024.
}

Our laboratory has prepared a number of conditionally -immortalized cell lines that appear to represent oligodendrocytes at different developmental stages in the lineage. One of these, N19, expresses the cell surface lipid markers GD3 and A2B5, characteric of oligodendrocyte precursors. The other line, N20.1, expresses the mRNAs for MBP and PLP as well as the cell-surface marker, galactocerebroside (Mab 01). While it expresses the MBP and PLP mRNAs, it does not express the proteins under our in vitro culture conditions. The N20.1 cell line, therefore, seems to represent a late immature/early mature cell in the oligodendrocyte lineage. Thus, the two lines represent cells at different stage of oligodendrocyte development.

Plasmid cDNA libraries were prepared and directional Tag PCR subtraction was used to generate cDNAs of RNAs that might be enriched in the N19 cell line. From this screen two small cDNA clones were isolated and used to screen a N19 cDNA library to obtain larger cDNA clones. On Northern blots each was highly enriched in the N19 cell line compared to the $\mathrm{N} 20.1$ cell line. One clone, designated $3 \mathrm{G} 5$, hybridized to an $\sim 0.6 \mathrm{~kb}$ mRNA, and was homologous to an uncharacterized, anonymous clone previously reported in a general screen of mouse embryonal carcinoma F9 cDNAs. The 640 bp 3G5 cDNA contained an open reading frame of 66 amino acids. We prepared a polyclonal antibody to 19 aa peptide within the predicted sequence. Combined in situ hybridizationimmunocytochemistry and double immunocytochemistry of mouse brain primary glial cell cultures showed co-expression of 3G5 in A2B5+ cells. The second cDNA hybridized to a $3.4 \mathrm{~kb}$ mRNA and encodes a cadherin, which is lacking a cytoplasmic domain. This cDNA and its encoded protein is highly homologous to chicken T-cadherin and human cadherin 13 (H-cadherin). In situ hybridization of mouse primary glial cells confirmed expression of this cadherin mRNA in oligodendrocyte-like cells. Double immunocytochemistry with a polyclonal antibody raised to a 31 amino-acid peptide showed colocalization with A2B5+ cells in mouse primary glial cultures. This cadherin has not previously been reported to be expressed in glial cells. Both 3G5 and the cadherin represent new candidates for stage-specific expression within cells of the oligodendrocyte lineage. (Supported by NIH grant NS23022 and MS Society grants PP0403 and RG2693). 


\title{
Abstracts for Posters
}

\section{P49}

\section{Specification of the oligodendrocyte lineage during murine embryonic CNS development.}

\author{
N.Spassky, C.Goujet-Zalc, C.Olivier, S.Martinez*, K.Ikenaka\#, B.Zalc and J-L.Thomas,. \\ INSERM U-134, Hôpital de la Salpêtrière, 75651 Paris cedex 13.* University of Murcia, \\ Spain, "NIPS, University of Okazaki, Japan.
}

We have previously described the pattern of expression of $d m-20$, an alternatively spliced transcript of the plp (proteolipid protein) gene, during embryonic development in the mouse. This description has led us to propose that $d m-20$ is expressed in oligodendrocyte precursors, which differentiate in restricted locations of the germinative neuroepithelium, starting from 9.5 days post conception. To test this hypothesis, we generated transgenic mice expressing a fusion protein made of a positive selection marker (product of the sh ble gene), which confers resistance to the antibiotic zeocin, and the E.coli lacZ reporter gene encoding $\beta$-galactosidase $(\beta$-gal), under the control of the promoter and regulator sequences of the plp gene (plp-sh blelac $Z$ ). Out of 6 transgenic lines obtained, 3 showed embryonic $\beta$-gal expression patterns superimposable to the pattern of endogenous $d m-20$. In the CNS of these animals, $\beta$-gal positive-cells never expressed markers of differentiated neurons (NeuroD transcript or type III $\beta$-tubulin). In vitro, as well as in vivo, the $\beta$-gal positive cells did not express markers of radial glia cells (RC2), astrocytes (GFAP) or neurons (type III $\beta$-tubulin, Hu, MAP-2). In contrast, the $\beta$-gal-positive cells coexpressed specific markers of the oligodendroglial lineage: A2B5 (progenitors), O4 (pre-oligodendrocytes), GalC and RIP (post-mitotic oligodendrocytes). The developmental potentialities of $\beta$-gal-positive cells were analysed in vitro by cultivating neuroepithelial cells isolated from E12.5 plp-sh ble-lacZ transgenic embryos in the presence of zeocin. After 15 days of treatment we observed an enrichment of the $\beta$-gal-positive/O4 positive cell population which represented $20 \%$ and $90 \%$ in controls and zeocin treated cultures, respectively. In addition, the pattern of $d m-20$ (or $\beta$-gal) expressing cells was discontinuous along the ventral neuroepithelium. $D m-20^{+}$cells were absent from the mesencephalic basal plate and rhombomers 3 to 5 . In contrast, $p d g f-\alpha$ receptor expressing cells were present in these 2 territories. Interestingly, cultures of the $3-\mathrm{r} 5$ region gave rise to $\mathrm{O4}^{+} / \beta$-gal-negative cells suggesting that these preoligodendrocyte cells may originate from $p d g f \alpha$ receptor expressing precursors and led us to propose the existence of at least 2 populations of oligodendrocyte precursors, each having a different location and timing of differentiation. The collective findings of our study indicate that the plp-sh ble-lacZ transgenic mouse should prove to be a power tool for the analysis of oligodendrocyte specification. 


\title{
Abstracts for Posters
}

P50

\section{GLIAL PROGENITOR CELLS IN DEVELOPING AND MATURE BRAIN IDENTIFIED BY ANTIBODIES TO NG2 PROTEOGLYCAN AND PDGF ALPHA RECEPTOR}

\author{
Akiko Nishiyama \\ Department of Neurosciences, Cleveland Clinic Foundation, Ohio, USA
}

We have been studying a population of glial cells in the rodent brain by using antibodies against the NG2 proteoglycan and the alpha receptor for platelet-derived growth factor (PDGF $\alpha$ receptor). In vitro the two molecules are expressed on the surface of A2B5-positive oligodendrocyte progenitor cells but are down-regulated as they differentiate into mature oligodendrocytes in serum-free medium. Our previous biochemical studies indicate that the two molecules are found in a multimolecular complex at the cell surface. Furthermore, we have shown that down-regulation of NG2 with anti-NG2 antibody diminishes the ability of the progenitor cells to proliferate in response to PDGF. In the developing rat brain in vivo antibodies to NG2 and PDGF $\alpha$ receptor label numerous process-bearing cells that share many characteristics previously reported for oligodendrocyte progenitor cells. Furthermore, the immunoreactive cells are present not only in the developing brain but are also found in large numbers distributed uniformly throughout the gray and white matter of the mature brain. The immunoreactive cells in both gray and white matter co-express NG2 and PDGF $\alpha$ receptor, suggesting that they are antigenically related to oligodendrocyte progenitor cells. Double-labeling of postnatal day 11 (P11) rat brain sections with antibodies to NG2 and proteolipid protein (PLP) or myelin basic protein (MBP) revealed that a small proportion of NG2+ cells expressed PLP or MBP, while the majority of the NG2+ cells did not express the myelin proteins, suggesting a transition from NG2+ progenitor cells into mature oligodendrocytes. Double-labeling of P30 and P90 rat brain sections with antibody to NG2 and GSA I-B 4 isolectin from Griffonia simplicifolia or antibody to glial fibrillary acidic protein revealed no doublelabeled cells, indicating that NG2+ cells are distinct from microglia or mature astrocytes. The NG2+/PDGF $\alpha$ receptor $+(\mathrm{N}+\mathrm{P}+)$ cells in the $\mathrm{P} 90$ brain display a complex morphology with highly branched processes, in contrast to the simple bipolar and oligopolar morphology of $\mathrm{N}+\mathrm{P}+$ cells in the embryonic CNS. Preliminary studies on the proliferative activity of NG2 + cells suggest that bromodeoxyuridine-labeling index of NG2+ cells declines from greater than $10 \%$ during the first postnatal week to less that $0.5 \%$ in the $\mathrm{P} 90$ brain. This suggests that the large population of $\mathrm{N}+\mathrm{P}+$ cells in the adult brain represent a relatively quiescent population of progenitor cells. We have observed that $\mathrm{N}+\mathrm{P}+$ cells increase in number and undergo morphological changes during the acute, inflammatory phase of experimental autoimmune encephalitis. Double-labeling studies indicate that the 'reactive NG2+ cells' are distinct from but are closely apposed to activated microglial cells, suggesting a functional interaction between the two cell types. 


\section{Abstracts for Posters}

\section{P51}

Reactive astrocytes and mossy fiber sprouting induced by kainic acid in rat hippocampus

Chunxi Han, Wang-Jie Yu, Hiroaki Asou and Keiichi Uyemura Department of Physiology, Keio University School of Medicine, Shinanomachi, Shinjuku-ku,Tokyo 160, Japan

Administration of kainic acid (KA), a glutamate receptor agonist, to rat induced morphological and biochemical changes in the rat brain, such as neuronal degeneration, reactive gliosis and sprouting of mossy fibers in hippocampus. Axonal sprouting and synaptic reorganization play an important role in the adaptation of the CNS to injury. However, the molecular mechanisms underlying this neural plasticity are poorly understood. Using the rat model of temporal lobe epilepsy induced by KA, we examined the relationship between mossy fiber sprouting and reactive astrocytes. Immunohistochemical stainings of glial fibrillary acidic protein (GFAP), intermediate filament protein vimentin and highly sialylated neural cell adhesion molecule (NCAM-H) were carried out after KA treatment. At 3 days after KA treatment, the neuronal cell degeneration and cell death occurred especially in CA1, where GFAP positive reactive astrocytes with the distinct processes were observed. By double stainings, the GFAP positive astrocytes were both vimentin and NCAM-H positive. The Timm staining demonstrated the sprouting of the mossy fibers only in the inner molecular layer of dentate gyrus in the present study. The appearance of reactive astrocytes with NCAM-H in the molecular layer of dentate gyrus are more remarkable in the rats with mossy fiber sprouting than that in the rats without sprouting. While NCAM-H expression was reported in newly generated granule cells in dentate gyrus of normal adult rat, reactive astrocytes induced KA also expressed NCAM-H. In the light of the known functional properties of NCAM-H, we suggest that the reactive astrocytes by means of the expression of NCAM-H may play a role in the sprouting of the mossy fibers in the inner molecular layer of the hippocampus following the systemic injection of KA. 


\title{
Abstracts for Posters
}

\section{P52}

\section{Treatment of Metastatic Brain Tumors with A Replication- Competent Multi-Mutated Herpes Simplex Virus-1}

\author{
Masahiro Toda 1, 2, 3, Keiichi Uyemura2, Takeshi Kawase3, Samuel \\ D. Rabkin 1, Robert L. Martuza 1 \\ 1 Georgetown Brain Tumor Center, Department of Neurosurgery, Georgetown \\ University Medical Center, 3800 Reservoir Road, NW, Washington, D.C. 20007 \\ USA and Departments of 2 Physiology and ${ }^{3}$ Neurosurgery , School of Medicine, \\ Keio University, 35 Shinanomachi, Shinjuku-ku, Tokyo 160, Japan.
}

We have created a replication-competent multi-gene mutant of HSV-1, designated G207, with deletions in the $\gamma 34.5$ gene and a lac $Z$ gene insertion in the ICP6 gene for brain tumor therapy. From a therapeutic perspective, G207 has advantages over other virus vectors. G207 replicates in dividing cells, undergoing a lytic infection with consequent cell death, however growth in nondividing cells is highly attenuated, targeting viral spread to the tumor. G207 is non-neuropathogenic, causing no detectable disease in mice and non-human primates. To investigate the therapeutic efficacy of G207 for the treatment of metastatic brain tumors, we developed two experimental models. One, an intracerebral xenograft tumor model with human breast adenocarcinoma in athymic mice and the second, a syngeneic tumor model with murine colon adenocarcinoma in BALB/c mice.

In vitro studies demonstrated that G207 efficiently destroyed three out of four human malignant breast cancer cell lines. MDA-MB-435 was most susceptible and MDA-MB-231 was least susceptible to replication by G207. In athymic mice harboring subcutaneous or intracerebral MDA-MB-435 cells, a single intraneoplastic inoculation of G207 caused growth inhibition and/or prolonged survival. In contrast, G207 had minimal effects on MDA-MB-231 subcutaneous tumor growth or survival in the intracerebral tumor model. The efficacy of G207 therapy in vivo correlated well with the susceptibility of the human cancer cells to G207 replication in vitro. Histological studies indicate that G207 replication is restricted to tumor cells in vivo and does not occur in the surrounding brain tissue.

In the syngeneic bilateral subcutaneous tumor model, intratumoral inoculation of G207 into established CT26 murine colon tumors caused a significant reduction in growth of both the infected tumors, as well as the contralateral non-inoculated tumors. Seven days after the virus inoculation, lac $Z$ expression from G207 was detected by X-gal histochemistry in the inoculated tumor, but not the non-inoculated tumor. The antitumor effect on the noninoculated contralateral tumor depended upon intratumoral inoculation of G207, as intradermal inoculation of G207 in the right flanks of mice with established unilateral tumors in the left flanks had no effect on tumor growth. Treatment of established CT26 subcutaneous tumors in athymic mice with similar doses of G207 did not reduce tumor growth. This suggests a role for T-cell mediated tumor immunity in the immune-competent mice. Therefore, G207 is able to inhibit local tumor growth by direct cytotoxic viral replication and induce a systemic antitumor immune response to inhibit metastatic tumors. 